\title{
BALANCED TOURNAMENT DESIGNS WITH ALMOST ORTHOGONAL RESOLUTIONS
}

\author{
E. R. LAMKEN and S. A. VANSTONE
}

(Received 29 April 1988; revised 4 August 1989)

Communicated by L. Caccetta

\begin{abstract}
A balanced tournament design, $B T D(n)$, defined on a $2 n$-set $V$ is an arrangement of the $\left(\begin{array}{c}2 n \\ 2\end{array}\right)$ distinct unordered pairs of the elements of $V$ into an $n \times 2 n-1$ array such that (1) every element of $V$ is contained in precisely one cell of each column, and (2) every element of $V$ is contained in at most two cells of each row. In this paper, we investigate the existence of balanced tournament designs with a pair of almost orthogonal resolutions. These designs can be used to construct doubly resolvable $(v, 3,2)-B I B D$ s and, in our smallest applications, have been used to construct previously unknown doubly resolvable $(v, 3,2)-B I B D \mathbf{s}$.
\end{abstract}

1980 Mathematics subject classification (Amer. Math. Soc.) (1985 Revision): 05 B 30.

\section{Introduction}

A balanced tournament design, $B T D(n)$, defined on a $2 n$-set $V$ is an arrangement of the $\left(\begin{array}{c}2 n \\ 2\end{array}\right)$ distinct unordered pairs of the elements of $V$ into an $n \times 2 n-1$ array such that

(1) every element of $V$ is contained in precisely one cell of each column, and

(2) every element of $V$ is contained in at most two cells of each row.

An example of a balanced tournament design, a $B T D(3)$, is displayed in Figure 1.

The existence of $B T D(n)$ s was established in [16]. (A simpler proof of this result appears in [7].)

(C) 1990 Australian Mathematical Society $0263-6115 / 90 \$ A 2.00+0.00$ 


\begin{tabular}{|c|c|c|c|c|}
\hline 12 & 15 & 45 & 24 & 03 \\
\hline 53 & 20 & 23 & 05 & 14 \\
\hline 04 & 34 & 01 & 13 & 25 \\
\hline
\end{tabular}

FIGURE 1. $B T D(3)$

THEOREM 1.1. For $n$ a positive integer, $n \neq 2$, there exists a $B T D(n)$.

An element which is contained only once in row $i$ of a $B T D(n)$ is called a deficient element of row $i$. The two deficient elements of row $i$ are referred to as the deficient pair of row $i$. It is easy to prove the following.

LEMMA 1.2. The deficient pairs of a $B T D(n)$ defined on a $2 n$ set $V$ partition the elements of $V$ into $n$ pairs.

A resolution of a $B T D(n)$ is a resolution of the $(2 n, 2,1)-B I B D$ contained in its cells. Let $B$ be a $B T D(n)$. Let $R_{1}, R_{2}, \ldots, R_{n}$ be the rows of $B$ and let $C_{1}, C_{2}, \ldots, C_{2 n-1}$ be the columns of $B . C=\left\{C_{1}, C_{2}, \ldots\right.$, $\left.C_{2 n-1}\right\}$ is a resolution of $B$. A resolution $D=\left\{D_{1}, D_{2}, \ldots, D_{2 n-1}\right\}$ will be called almost orthogonal to $C$ if

(i) $C_{2 n-1}=D_{2 n-1}$,

(ii) $\left|C_{i} \cap D_{j}\right| \leq 1$ for $1 \leq i, j \leq 2 n-2$,

(iii) $\left|D_{j} \cap R_{i}\right|=1$ for $j=1,2, \ldots, 2 n-1$ and $i=1,2, \ldots, n$.

If $D$ exists, we say that $B$ has a pair of almost orthogonal resolution (AORs). The columns of the array form one resolution and the columns of the array in Figure 2 form an almost orthogonal resolution.

\begin{tabular}{|c|c|c|c|c|}
\hline 12 & 15 & 45 & 24 & 03 \\
\hline 05 & 23 & 20 & 53 & 14 \\
\hline 34 & 04 & 13 & 01 & 25 \\
\hline
\end{tabular}

FIGURE 2. $B T D(3)$

Let $B$ be a $B T D(n)$ with $A O R$ s. Suppose that the resolution class which is shared by the pair of almost orthogonal resolutions contains the deficient pairs of $B$. Then $B$ is said to have Property $C^{\prime}$. As an example, the $B T D(3)$ in Figures 1 and 2 has Property $C^{\prime}$.

In several previous papers, we have described new constructions for balanced tournament designs, factored balanced tournament designs (FBTDs), and partitioned balanced tournament designs (PBTDs) [7-10]. The original study of $B T D(n)$ s [16] was motivated by their tournament scheduling applications. The designs have become of interest more recently because of their 
relationships to several other types of combinatorial designs, such as Room squares and Howell designs. In this paper, we investigate the existence of balanced tournament designs with a pair of almost orthogonal resolutions. In [11], we describe an application of these designs to constructing doubly resolvable $(v, 3,2)-B I B D$ s. Examples of these constructions can be found in [6]. In the smallest cases, we have been able to use balanced tournament designs with $A O R$ s to construct previously unknown $D R(v, 3,2)-B I B D \mathrm{~s}$. Thus, determining the spectrum of balanced tournament designs with $A O R \mathrm{~s}$ would provide new $D R(v, 3,2)-B I B D$ s.

In Section 2, we describe direct constructions and constructions which use Howell designs of side $2 n$ and order $2 n+2, H(2 n, 2 n+2)$, for balanced tournament designs with $A O R \mathrm{~s}$. There are two basic frame constructions for balanced tournament designs with $A O R \mathrm{~s}$; these use complementary frames with Property $T$ and frames with Property T2. We define the frames and describe the constructions in Section 3. Existence results for complementary frames with property $T$ and frames with Property $T 2$ are provided in Section 4. Finally, we apply our constructions in the last section to prove the existence of several infinite classes of balanced tournament designs with $A O R \mathrm{~s}$.

\section{Direct constructions and Howell design constructions}

In this section, we use Howell designs of side $2 n$ and order $2 n+2$, $H(2 n, 2 n+2)$, to construct balanced tournament designs with $A O R \mathrm{~s}$.

Let $V$ be a set of $2 n$ elements. A Howell design of side $s$ and order $2 n$, or more briefly an $H(s, 2 n)$, is an $s \times s$ array in which each cell is either empty or contains an unordered pair of elements from $V$ such that

(1) each row and each column is Latin (that is, every element of $V$ is in precisely one cell of each row and column), and

(2) every unordered pair of elements of $V$ is in at most once cell of the array. It follows immediately from the definition of an $H(s, 2 n)$ that $n \leq s \leq 2 n-1$. The spectrum of Howell designs was determined by $\mathrm{D}$. R. Stinson in [17].

THEOREM 2.1 [16]. There exists an $H(s, 2 n)$ if and only if $(2 n, s)$ satisfies $n \leq s \leq 2 n-1$ and $(s, 2 n) \notin\{(3,4),(2,4),(6,6),(5,8)\}$.

Our first construction is a starter-adder construction for a $B T D(n+1)$. We will use a starter for an $H(2 n, 2 n+2)$. For definitions and results on starters and adders for $H(2 n, 2 n+2)$, we refer to [1]. 
THEOREM 2.2. Let $S=\left\{\left\{x_{1}, y_{1}\right\},\left\{x_{2}, y_{2}\right\}, \ldots,\left\{x_{n-1}, y_{n-1}\right\},\left\{\alpha, z_{1}\right\}\right.$, $\left.\left\{\infty, z_{2}\right\}\right\}$ be a starter for an $H(2 n, 2 n+2)$ defined on $Z_{2 n} \cup\{\alpha, \infty\}$. Let $S^{\prime}=S-\left\{x_{i}, y_{i}\right\}$ for some $i, 1 \leq i \leq n-1$. Suppose there is an adder $A=\left(a_{1}, a_{2}, \ldots, a_{n}\right)$ of $n$ distinct elements of $Z_{2 n}$ such that

(i) $\left|a_{k}-a_{j}\right| \neq n$ for $1 \leq i, j \leq n$, and

(ii) $\left(S^{\prime}+A\right) \cup\left(S^{\prime}+(A+n)\right)$ contains every element of $Z_{2 n} \cup\{\alpha, \infty\}$ at most twice.

Then there is a $B T D(n+1)$.

Proof. Let $S^{\prime}=\left(B_{1}, B_{2}, \ldots, B_{n}\right)$ and let $A=\left(a_{1}, a_{2}, \ldots, a_{n}\right)$. The pair which has been removed from $S$ is $\left\{x_{i}, y_{i}\right\}$ and the pairs which do not occur in the $H(2 n, 2 n+2)$ are $\{\alpha, \infty\},\{0, n\},\{1, n+1\}, \ldots,\{n-1,2 n-$ $1\}$. Let $\left(S^{\prime}+A\right) \cup\left(S^{\prime}+(A+n)\right)=2\left(Z_{2 n} \cup\{\alpha, \infty\}\right)-\left(\left\{u_{1}, v_{1}\right\} \cup\left\{u_{2}, v_{2}\right\}\right)$ where $\left|u_{i}-v_{i}\right|=n$ for $i=1,2$.

We construct an $n+1 \times 2 n+1$ array $B$ defined on $Z_{2 n} \cup\{\alpha, \infty\}$ as follows. Label the rows of $B 0,1,2, \ldots, n$ and the columns of $B 0,1,2, \ldots, 2 n$. For $i=1,2, \ldots, n$ and $j=0,1,2, \ldots, n-1$, place $B_{i}+a_{i}+j$ in cell $\left(j, a_{i}+j\right)$ and place $B_{i}+a_{i}+n+j$ in cell $\left(j, a_{i}+n+j\right)$. (The second argument is taken modulo $2 n$.) In row $n$ and column $j$ place $\left\{x_{i}+j, y_{i}+j\right\}$ for $j=0,1, \ldots, 2 n-1$. In row $j$ and column $2 n$ place $\left\{u_{1}+j, v_{1}+j\right\}$ for $j=0,1, \ldots, n-1$. Finally, in cell $(n, 2 n)$ place the pair $\{\alpha, \infty\}$.

The resulting array $B$ is a $B T D(n+1)$ defined on $Z_{2 n} \cup\{\alpha, \infty\}$. The first column of $B$ contains the pairs in $S$. Column $j$ of $B$ contains the pairs in $S+j$ for $j=0,1, \ldots, 2 n-1$. Column $2 n$ of $B$ contains the pairs $\{\alpha, \infty\}$, $\{0, n\},\{1, n+1\}, \ldots,\{n-1,2 n-1\}$. Thus, each column of $B$ contains every element of $Z_{2 n} \cup\{\alpha, \infty\}$ precisely once. The adder $A$ is defined so that every element of $Z_{2 n} \cup\{\alpha, \infty\}$ occurs at most twice in each row of $B$. The deficient pair for row $j$ is $\left\{u_{2}+j, v_{2}+j\right\}$ for $j=0,1, \ldots, n-1$ and $\{\alpha, \infty\}$ for row $n$. Since we have used a starter for an $H(2 n, 2 n+2)$ and added the pairs which did not occur, every distinct pair in $Z_{2 n} \cup\{\alpha, \infty\}$ occurs once in $B$. This verifies that $B$ is a $B T D(n+1)$.

This construction was modified in $[7,8]$ to produce factored $B T D \mathrm{~s}$ $(F B T D \mathrm{~s})$ and partitioned $B T D \mathrm{~s}$ (PBTD s). The following corollary describes how to use this starter-adder construction to produce balanced tournament designs with $A O R \mathrm{~s}$.

Corollary 2.3. Let $B$ be a $B T D(n+1)$ constructed using Theorem 2.2. Let $\left|x_{i}-y_{i}\right|=\Delta$ and let $T=\left(S^{\prime}+A\right) \cup\left(S^{\prime}+(A+n)\right)$. Let $T^{\prime}=$ $\left\{T_{1}, T_{2}, \ldots, T_{n}\right\}$ be a set of $n$ pairs chosen from $T$ such that every difference in $Z_{2 n}$ except $n$ and $\Delta$ occurs once and such that the elements $\alpha$ and $\infty$ 
each occur once in $T^{\prime}$. Suppose there is an adder $A^{\prime}=\left(a_{1}^{\prime}, a_{2}^{\prime}, \ldots, a_{n}^{\prime}\right)$ of $n$ distinct elements of $Z_{2 n}$ such that

(i) $\left|a_{k}^{\prime}-a_{j}^{\prime}\right| \neq n$ for $1 \leq i, j \leq n$,

(ii) $Z_{2 n} \cup\{\alpha, \infty\}-\left(T^{\prime}+A^{\prime}\right)=\{r, t\}$ where $|r-t|=\Delta$,

(iii) $\left(S^{\prime}+c\right) \cap\left(T^{\prime}+A^{\prime}\right)=\varnothing$ where $\left\{x_{i}+c, y_{i}+c\right\}=\{r, t\} .\left(T^{\prime}+A^{\prime}\right.$ and $S^{\prime}+c$ are sets of pairs here.)

Then $B$ has a pair of almost orthogonal resolutions.

Proof. The columns $C_{1}, C_{2}, \ldots, C_{2 n+1}$ form one resolution for the $B T D(n+1)$. The adder $A^{\prime}$ is defined so that an almost orthogonal resolution contains $C_{2 n+1}$ and the resolution classes $R_{1}, R_{2}, \ldots, R_{2 n}$ where

$$
R_{i}=\left(T^{\prime}+A^{\prime}+(i-1)\right) \cup\{r+i-1, t+i-1\}, \quad 1 \leq i \leq 2 n .
$$

The smallest non-trivial $B T D$ with $A O R \mathrm{~s}$ is displayed in Figures 1 and 2. This design could also be described using starters and adders. Since there do not exist starters and adders for $H(6,8)$ and $H(8,10)$ [5], we illustrate this theorem for the next case, using a starter-adder pair for an $H(10,12)$.

LEMMA 2.4. There exists a BTD(6) with AOR s.

Proof. We list the starters and adders below. The array is displayed in Figure 3.

$$
\begin{aligned}
& S^{\prime} \quad 12040469 \quad \alpha 7 \quad \infty 8 \quad\left\{u_{1}, v_{1}\right\}=\{0,5\} \\
& \text { A } \begin{array}{rrrrrr}
3 & 7 & 0 & 6 & 4 \\
\hline 45 & 71 & 69 & \alpha 3 & \infty 2
\end{array} \quad\left\{u_{2}, v_{2}\right\}=\{3,8\} \\
& \left\{x_{i}, y_{i}\right\}=\{3,5\} \\
& \begin{array}{lllllll}
T^{\prime} & 69 & 71 & 45 & \alpha 3 & \infty & 2
\end{array}
\end{aligned}
$$

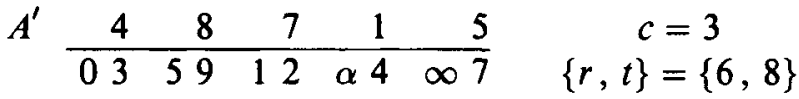

The next construction in this section uses the equivalence between $B T D \mathrm{~s}$ with $A O R$ s and $H(2 n, 2 n+2)$ s with Property $\mathrm{T} 2$.

Let $H$ be an $H(2 n, 2 n+2)$ defined on a set $V=\left\{x_{i}, y_{i} \mid 1 \leq i \leq n+\right.$ 1\}. Suppose the pairs which do not occur in $H$ are $\left\{x_{i}, y_{i}\right\}$ for $1 \leq i \leq$ $n+1$; and let $M$ denote this set of pairs. We say $H$ has Property T2 if we can partition the nonempty cells of $H$ into $n+1$ transversals of $2 n$ cells each, $T_{1}, T_{2}, \ldots, T_{n+1}$, such that (1) every element in $V$ occurs at most twice in the pairs in $T_{i}, 1 \leq i \leq n+1$, and (2) the four elements $u_{i 1}, u_{i 2}, v_{i 1}, v_{i 2}$ which occur at most once each in $T_{i}$ can be used to form 
two pairs $\left\{u_{i 1}, v_{i 1}\right\}$ and $\left\{u_{i 2}, v_{i 2}\right\}$ where $\left\{\left\{u_{i 1}, v_{i 1}\right\} \mid i=1,2, \ldots, n+1\right\}=$ $M$ and $\bigcup_{i=1}^{n+1}\left\{u_{i 2}, v_{i 2}\right\}=V$.

\begin{tabular}{|l|l|l|l|l|l|l|l|l|l|l|}
\hline 69 & $\alpha 8$ & 26 & 45 & $\infty 2$ & 14 & $\alpha 3$ & 71 & 90 & $\infty 7$ & 05 \\
\hline$\infty 8$ & 70 & $\alpha 9$ & 37 & 56 & $\infty 3$ & 25 & $\alpha 4$ & 82 & 01 & 16 \\
\hline 12 & $\infty 9$ & 81 & $\alpha 0$ & 48 & 67 & $\infty 4$ & 36 & $\alpha 5$ & 93 & 27 \\
\hline 04 & 23 & $\infty 0$ & 92 & $\alpha 1$ & 59 & 78 & $\infty 5$ & 47 & $\alpha 6$ & 38 \\
\hline$\alpha 7$ & 15 & 34 & $\infty 1$ & 03 & $\alpha 2$ & 60 & 89 & $\infty 6$ & 58 & 49 \\
\hline 35 & 46 & 57 & 68 & 79 & 80 & 91 & 02 & 13 & 24 & $\alpha \infty$ \\
\hline
\end{tabular}

FIGURE 3. $B T D(6)$ with $A O R \mathrm{~s}$

THEOREM 2.5. There exists a BTD $(n+1)$ with a pair of almost orthogonal resolutions if and only if there is an $H(2 n, 2 n+2)$ with Property T2.

Proof. Let $B$ be a $B T D(n+1)$ with $A O R$ s defined on a set $V$. The columns $C_{1}, C_{2}, \ldots, C_{2 n+1}$ form one resolution of the design. Suppose the almost orthogonal resolution has resolution classes $B_{1}, B_{2}, \ldots, B_{2 n+1}$ where $B_{2 n+1}=C_{2 n+1}$. Let $R_{1}, R_{2}, \ldots, R_{n+1}$ be the rows of $B$.

We construct an $H(2 n, 2 n+2), H$ from $B$ by using the pair of almost orthogonal resolutions. Index the rows of $H$ by the resolution classes $B_{1}, B_{2}, \ldots, B_{2 n}$ and the columns by $C_{1}, C_{2}, \ldots, C_{2 n}$. If $B_{i} \cap C_{j} \neq \varnothing$, place $B_{i} \cap C_{j}$ in cell $\left(B_{i}, C_{j}\right)$. The pairs in $R_{i}$ with the pair in $C_{2 n+1}$ deleted will form a transversal $T_{i}$ of $H$. Every element in $V$ will occur at most twice and the pair in $C_{2 n+1}$ will not occur in $H$. Thus, the rows of $B$ correspond to a set of $n+1$ transversals of $H$ and $H$ has Property T2.

Conversely, if $H$ has Property T2, we use the transversals to form $n+1$ rows of an array $B$. Use the columns of $H$ to form the columns of the array $B$ and add a last column with the pairs $\left\{u_{i 1}, v_{i 1}\right\}$ for $1 \leq i \leq n+1$. The rows of $H$ will form an almost orthogonal resolution (the last column will be the same in each resolution). The deficient pairs will be $\left\{u_{i 2}, v_{i 2}\right\}$ for $i=1,2, \ldots, n+1$.

We use Theorem 2.5 to provide results for the next two smallest cases, $B T D(n)$ for $n=4$ and 5 .

LEMMA 2.6. There does not exist a $B T D(4)$ with a pair of almost orthogonal resolutions.

Proof. There exist three non-isomorphic Howell designs $H(6,8)$ [15]. We show that none of these designs has Property T2. 
(i) Let

$$
H_{1}=\left[\begin{array}{llllll}
13 & 68 & & 24 & & 57 \\
67 & 14 & & 58 & & 23 \\
48 & & 15 & 37 & 26 & \\
25 & & 47 & 16 & 38 & \\
& 35 & 28 & & 17 & 46 \\
& 27 & 36 & & 45 & 18
\end{array}\right] .
$$

The pairs which do not occur in $H_{1}$ are $\{1,2\},\{3,4\},\{5,6\}$, and $\{7,8\}$. There are three possible transversals of $H_{1}$ which contain the pair $\{1,3\}$. These are

$$
\begin{array}{llllll}
13 & 35 & 58 & 26 & 47 & 18 \\
13 & 27 & 58 & 46 & 15 & 38 . \\
13 & 27 & 58 & 46 & 26 & 47
\end{array}
$$

Since none of these transversals is missing a pair which does not occur in $H_{1}, H_{1}$ cannot have Property $\mathrm{T} 2$.

(ii) Let

$$
H_{2}=\left[\begin{array}{llllll}
13 & 57 & 68 & 24 & & \\
67 & 14 & 23 & 58 & & \\
48 & & 15 & & 36 & 27 \\
& & 47 & 16 & 28 & 35 \\
25 & 38 & & & 17 & 46 \\
& 26 & & 37 & 45 & 18
\end{array}\right] .
$$

The pairs which do not occur in $H_{2}$ are $\{1,2\},\{3,4\},\{5,6\}$ and $\{7,8\}$. There are two possible transversals which contain $\{1,3\}$. These are

$$
\begin{array}{llllll}
13 & 38 & 47 & 27 & 58 & 45 \\
13 & 26 & 15 & 28 & 46 & 58
\end{array} .
$$

If $H_{2}$ is to satisfy Property T2, then the first transversal must have $\left\{u_{11}, v_{11}\right\}$ $=\{1,2\}$ and the second must have $\left\{u_{11}, v_{11}\right\}=\{3,4\}$. But, this forces $u_{12}=v_{12}$ in both cases. So $H_{2}$ cannot have Property T2.

(iii) Let

$$
H_{3}=\left[\begin{array}{rrrrrr}
13 & 68 & & 24 & & 57 \\
67 & 14 & & 58 & & 23 \\
48 & 27 & 15 & & 36 & \\
& 35 & 47 & 16 & 28 & \\
25 & & 38 & & 17 & 46 \\
& & 26 & 37 & 45 & 18
\end{array}\right] .
$$

There is no transversal of $H_{3}$ which contains the pair $\{6,7\}$, and so $H_{3}$ cannot have Property T2.

LEMMA 2.7. There exists a BTD(5) with a pair of almost orthogonal resolutions. 
Proof. The $B T D(5)$ in Figure 4 was constructed from the $H(8,10)$ in [12, Figure 1]. The columns of the arrays displayed in Figure 4 and Figure 5 form a pair of almost orthogonal resolutions for a $B T D(5)$.

\begin{tabular}{|l|c|c|c|c|c|c|c|c|}
\hline$\overline{2} \overline{3}$ & $\infty 1$ & $\infty 2$ & $\overline{0} \overline{1}$ & $\alpha 3$ & $3 \overline{2}$ & $0 \overline{1}$ & $\alpha 0$ & $\overline{0} \overline{3}$ \\
\hline 13 & $\alpha \overline{1}$ & $\alpha \overline{2}$ & 02 & $\infty \overline{0}$ & $1 \overline{3}$ & $\overline{0} 2$ & $\infty \overline{3}$ & 03 \\
\hline$\infty 0$ & $\overline{0} \overline{2}$ & $\overline{1} \overline{3}$ & $\infty 3$ & $0 \overline{2}$ & $\alpha 2$ & $\alpha 1$ & $3 \overline{1}$ & 12 \\
\hline$\alpha \overline{0}$ & 23 & 10 & $\alpha \overline{3}$ & $2 \overline{3}$ & $\infty \overline{1}$ & $\infty \overline{2}$ & $1 \overline{0}$ & $\overline{1} \overline{2}$ \\
\hline$\overline{1} 2$ & $\overline{3} 0$ & $\overline{0} 3$ & $\overline{2} 1$ & $1 \overline{1}$ & $0 \overline{0}$ & $3 \overline{3}$ & $2 \overline{2}$ & $\alpha \infty$ \\
\hline
\end{tabular}

Figure 4. A $B T D(5)$ with $A O R \mathrm{~s}$

\begin{tabular}{|l|c|c|c|c|c|c|c|c|}
\hline$\alpha 0$ & $\overline{2} \overline{3}$ & $\overline{0} \overline{1}$ & $\alpha 3$ & $3 \overline{2}$ & $\infty 1$ & $\infty 2$ & $0 \overline{1}$ & $\overline{0} \overline{3}$ \\
\hline$\infty \overline{0}$ & 02 & 13 & $\infty \overline{3}$ & $\overline{0} 2$ & $\alpha \overline{2}$ & $\alpha \overline{1}$ & $1 \overline{3}$ & 03 \\
\hline$\overline{1} \overline{3}$ & $\alpha 1$ & $\alpha 2$ & $\overline{0} \overline{2}$ & $\infty 0$ & $3 \overline{1}$ & $0 \overline{2}$ & $\infty 3$ & 12 \\
\hline 23 & $\infty \overline{1}$ & $\infty \overline{2}$ & 01 & $\alpha \overline{3}$ & $2 \overline{3}$ & $1 \overline{0}$ & $\alpha \overline{0}$ & $\overline{1} \overline{2}$ \\
\hline$\overline{2} 1$ & $\overline{0} 3$ & $\overline{3} 0$ & $\overline{1} 2$ & $1 \overline{1}$ & $0 \overline{0}$ & $3 \overline{3}$ & $2 \overline{2}$ & $\alpha \infty$ \\
\hline
\end{tabular}

FIGURE 5. A $B T D(5)$ whose columns form an almost orthogonal resolution for the $B T D(5)$ in Figure 4

\section{Frame constructions}

In this section, we describe frame constructions for balanced tournament designs with almost orthogonal resolutions. There are two basic constructions. The first one uses complementary frames with Property $T$ and sets of orthogonal partitioned incomplete Latin squares to construct balanced tournament designs with $A O R \mathrm{~s}$. The second uses frames with Property T2 to construct Howell designs with Property T2. In order to describe these constructions, we require several definitions.

Let $V$ be a set of $v$ elements. Let $G_{1}, G_{2}, \ldots, G_{m}$ be a partition of $V$ into $m$ sets. A $\left\{G_{1}, G_{2}, \ldots, G_{m}\right\}$-frame $F$ with block size $k$, index $\lambda$, and latinicity $\mu$ is a square array of side $v$ which satisfies the properties listed below. We index the rows and columns of $F$ by the elements of $V$.

(1) Each cell is either empty or contains a $k$-subset of $V$.

(2) Let $F_{i}$ be the subsquare of $F$ indexed by the elements of $G_{i}, F_{i}$ is empty for $i=1,2, \ldots, m$. (The subsquares of $F_{i}$ are often referred to as the holes of $F$.) 
(3) Let $j \in G_{i}$. Row $j$ of $F$ contains each element of $V-G_{i} \mu$ times and column $j$ of $F$ contains each element of $V-G_{i} \mu$ times.

(4) The collection of blocks obtained from the nonempty cells of $F$ is a $G D D\left(v ; k ; G_{1}, G_{2}, \ldots, G_{m} ; 0, \lambda\right)$. (See [21] for the notation for group divisible designs (GDDs).)

If there is a $\left\{G_{1}, G_{2}, \ldots, G_{m}\right\}$-frame $H$ with block size $k$, index $\lambda$, and latinicity $\mu$ such that

(1) $H_{i}=F_{i}$ for $i=1,2, \ldots, m$, and

(2) $H$ can be written in the empty cells of $F-\bigcup_{m}^{i=1} F_{i}$,

then $H$ is called a complement of $F$ and denoted by $\bar{F}$. If a complement of $F$ exists, we call $F$ a complementary $\left\{G_{1}, G_{2}, \ldots, G_{m}\right\}$-frame. A complementary $\left\{G_{1}, G_{2}, \ldots, G_{m}\right\}$-frame $F$ is said to be skew if at most one of the cells $(i, j)$ and $(j, i)(i \neq j)$ is nonempty.

We will use the following notation for frames. If $\left|G_{i}\right|=h$ for $i=$ $1,2, \ldots, m$, we call $F$ a $(\mu, \lambda ; k, m, h)$-frame. The type of a $\left\{G_{1}, G_{2}\right.$, $\left.\ldots, G_{m}\right\}$-frame is the multiset $\left\{\left|G_{1}\right|,\left|G_{2}\right|, \ldots,\left|G_{m}\right|\right\}$. We will say that a frame has type $t_{1}^{u_{1}} t_{2}^{u_{2}} \cdots t_{k}^{u_{k}}$ if there are $u_{i} G_{j}$ 's of cardinality $t_{i}, 1 \leq i \leq k$. A frame of type $t^{n}$ is called uniform, since all the holes have the same size. In this paper, we will restrict our attention to frames with $\mu=\lambda=1$ and $k=2$. For notational convenience, if $\mu=\lambda=1$ and $k=2$, we will denote a frame simply by its type or partitioning $\left(\left\{G_{1}, G_{2}, \ldots, G_{m}\right\}\right)$.

Let $V=\bigcup_{i=1}^{m} V_{i}$ and let $W=\bigcup_{i=1}^{m} W_{i}$ where $\left|V_{i}\right|=\left|W_{i}\right|$ for $i=$ $1,2, \ldots, m$. Let $F$ be a complementary $\left\{V_{1}, V_{2}, \ldots, V_{m}\right\}$-frame defined on $V$. Let $\bar{F}$ be a complement of $F$ defined on $W$ so that $\bar{F}$ is a $\left\{W_{1}, W_{2}, \ldots, W_{m}\right\}$-frame. $A$ will denote the array of pairs formed by the superposition of $F$ and $\bar{F}, A=F \circ \bar{F}$. We say that the complementary frame $F$ has Property $\mathrm{T}$ if there exists a set of $\sum_{i=1}^{m}\left|V_{i}\right|$ transversals, $T=\left\{T_{1}^{i}, T_{2}^{i}, \ldots, T_{\left|V_{i}\right|}^{i} \mid i=1,2, \ldots, m\right\}$, of the array $A$ such that

(i) $T_{k}^{i} \cap T_{i}^{j}=\varnothing$ for $1 \leq k \leq\left|V_{i}\right|, 1 \leq l \leq\left|V_{j}\right|, 1 \leq i, j \leq m$ excluding the case $k=l$ and $i=j$,

(ii) Every element of $\left(V-V_{i}\right) \cup\left(W-W_{i}\right)$ occurs precisely once in $T_{j}^{i}$ for $j=1,2, \ldots,\left|V_{i}\right|,(i=1,2, \ldots, m)$,

(iii) $T_{j}^{i}$ contains $\left|V_{i}\right|$ empty cells from the hole $F_{i}$.

We call $T$ a set of skew transversals if each transversal $T_{j}^{i}$ has the property that if cell $(x, y)$ is in the transversal then so is cell $(y, x)$. An example of a skew $1^{7}$ frame is displayed in Figure 6. This frame has a set of 7 skew transversals. The pairs contained in these transversals are listed in Table 1. The elements of the complement are not shown. 
Let $V=\bigcup_{i=1}^{m} V_{i}$ where $\left|V_{i}\right|=2 t_{i} \quad\left(t_{i}\right.$ is a positive integer $)$ for $i=$ $1,2, \ldots, m$. Suppose $F$ is a $\left\{V_{1}, V_{2}, \ldots, V_{m}\right\}$-frame defined on $V$. We say $F$ has Property $\mathrm{T} 2$ if there exists a set of $\sum_{i=1}^{m} t_{i}$ transversals, $\left\{T_{1}^{i}, T_{2}^{i}\right.$, $\left.\ldots, T_{t_{i}}^{i} \mid i=1,2, \ldots, m\right\}$, of $F$ such that

(i) $T_{k}^{i} \cap T_{l}^{i}=\varnothing$ for $1 \leq i, j \leq m, 1 \leq k \leq t_{i}, 1 \leq l \leq t_{j}$ excluding the case $i=j$ and $k=l$,

(ii) every element of $V-V_{i}$ occurs precisely twice in $T_{j}^{i}$ for $j=1,2, \ldots$, $t_{i}(i=1,2, \ldots, m)$,

(iii) $T_{j}^{i}$ contains $2 t_{i}$ empty cells from the hole $F_{i}$.

\begin{tabular}{|l|l|l|l|l|l|l|}
\hline & 26 & 45 & & 13 & & \\
\hline & & 30 & 56 & & 24 & \\
\hline & & & 41 & 60 & & 35 \\
\hline 42 & & & & 52 & 01 & \\
\hline & 50 & & & & 63 & 12 \\
\hline 23 & & 61 & & & & 04 \\
\hline 15 & 34 & & 02 & & & \\
\hline
\end{tabular}

Figure 6. A skew $1^{7}$ frame

TABLE 1. A set of skew transversals for Figure 6

\begin{tabular}{|l|l|l|l|}
\hline$T_{1}$ & 34 & 16 & 25 \\
\hline$T_{2}$ & 45 & 20 & 36 \\
\hline$T_{3}$ & 56 & 31 & 40 \\
\hline$T_{4}$ & 60 & 42 & 51 \\
\hline$T_{5}$ & 01 & 53 & 62 \\
\hline$T_{6}$ & 12 & 64 & 03 \\
\hline$T_{7}$ & 23 & 05 & 14 \\
\hline
\end{tabular}

An example of a $2^{5}$ frame with Property T2 is displayed in Figure 7. The five transversals are listed by their pairs in the frame in Table 2 .

In the next section, we discuss existence results for complementary frames with Property $\mathrm{T}$ and frames with Property T2.

We will also use sets of orthogonal partitioned incomplete Latin squares (OPILs) in the first construction. For completeness, we include the necessary definitions. We refer to $[3,19,20]$ for the existence results that we will use and for further information on $O P I L \mathrm{~s}$.

Let $P=\left\{S_{1}, S_{2}, \ldots, S_{m}\right\}$ be a partition of a set $S(m \geq 2)$. A partitioned incomplete Latin square, having partition $P$, is an $|S| \times|S|$ array $L$, 


\begin{tabular}{|c|c|c|c|c|c|c|c|c|c|}
\hline & & $\begin{array}{ll}2 & 1 \\
4 & 1\end{array}$ & & & $\begin{array}{ll}4 & 0 \\
3 & 1\end{array}$ & $\begin{array}{ll}1 & 0 \\
2 & 0\end{array}$ & & & $\begin{array}{ll}3 & 0 \\
1 & 1\end{array}$ \\
\hline & & & $\begin{array}{ll}20 \\
40\end{array}$ & $\begin{array}{lll}4 & 1 \\
3 & 0 \\
\end{array}$ & & & $\begin{array}{lll}1 & 1 \\
2 & 1\end{array}$ & $\begin{array}{ll}3 & 1 \\
1 & 0\end{array}$ & \\
\hline & $\begin{array}{ll}4 & 0 \\
2 & 1\end{array}$ & & & $\begin{array}{ll}3 & 1 \\
0 & 1\end{array}$ & & & $\begin{array}{ll}0 & 0 \\
4 & 1\end{array}$ & $\begin{array}{ll}2 & 0 \\
3 & 0\end{array}$ & \\
\hline $\begin{array}{lll}4 & 1 \\
2 & 0\end{array}$ & & & & & $\begin{array}{ll}3 & 0 \\
0 & 0\end{array}$ & $\begin{array}{lll}0 & 1 \\
4 & 0\end{array}$ & & & $\begin{array}{ll}2 & 1 \\
3 & 1\end{array}$ \\
\hline $\begin{array}{l}30 \\
40 \\
\end{array}$ & & & $\begin{array}{ll}00 \\
31 \\
\end{array}$ & & & $\begin{array}{ll}41 \\
11 \\
\end{array}$ & & & $\begin{array}{ll}1 & 0 \\
0 & 1 \\
\end{array}$ \\
\hline & $\begin{array}{ll}3 & 1 \\
4 & 1\end{array}$ & $\begin{array}{ll}0 & 1 \\
3 & 0\end{array}$ & & & & & $\begin{array}{l}40 \\
10\end{array}$ & $\begin{array}{ll}1 & 1 \\
0 & 0\end{array}$ & \\
\hline & $\begin{array}{ll}2 & 0 \\
11 & 1 \\
\end{array}$ & $\begin{array}{ll}4 & 0 \\
0 & 0 \\
\end{array}$ & & & $\begin{array}{ll}1 & 0 \\
4 & 1 \\
\end{array}$ & & & $\begin{array}{lll}0 & 1 \\
2 & 1\end{array}$ & \\
\hline $\begin{array}{ll}21 & 1 \\
1 & 0 \\
\end{array}$ & & & $\begin{array}{lll}4 & 1 \\
0 & 1 \\
\end{array}$ & $\begin{array}{ll}1 & 1 \\
4 & 0 \\
\end{array}$ & & & & & $\begin{array}{l}00 \\
20 \\
\end{array}$ \\
\hline $\begin{array}{lll}1 & 1 \\
3 & 1 \\
\end{array}$ & & & $\begin{array}{ll}3 & 0 \\
2 & 1 \\
\end{array}$ & $\begin{array}{ll}0 & 0 \\
10 & 0 \\
\end{array}$ & & & $\begin{array}{lll}2 & 0 \\
0 & 1 \\
\end{array}$ & & \\
\hline & $\begin{array}{ll}1 & 0 \\
3 & 0\end{array}$ & $\begin{array}{ll}3 & 1 \\
2 & 0\end{array}$ & & & $\begin{array}{ll}0 & 1 \\
1 & 1\end{array}$ & $\begin{array}{ll}2 & 1 \\
0 & 0\end{array}$ & & & \\
\hline
\end{tabular}

FIgURe 7. A $2^{5}$ frame with Property T2

TABle 2. Transversals for Property T2 of the $2^{5}$ frame in Figure 7

$\begin{array}{ccccccccc}T_{1}^{1}: & 31,20 & 30,21 & 11,40 & 10,41 & 20,30 & 21,31 & 41,11 & 40,10 \\ T_{1}^{2}: & 41,30 & 40,31 & 21,00 & 20,01 & 30,40 & 31,41 & 01,21 & 00,20 \\ T_{1}^{3}: & 01,40 & 00,41 & 31,10 & 30,11 & 40,00 & 41,01 & 11,31 & 10,30 \\ T_{1}^{4}: & 11,00 & 10,01 & 41,20 & 40,21 & 00,10 & 01,11 & 21,41 & 20,40 \\ T_{1}^{5}: & 21,10 & 20,11 & 01,30 & 00,31 & 10,20 & 11,21 & 31,01 & 30,00\end{array}$

indexed by the elements of $S$, satisfying the following properties.

(1) A cell of $L$ either contains an element of $S$ or is empty.

(2) The subarrays indexed by $S_{i} \times S_{i}$ are empty for $1 \leq i \leq m$.

(3) Let $j \in S_{i}$. Row $j$ of $L$ contains each element of $S-S_{i}$ precisely once and column $j$ of $L$ contains each element of $S-S_{i}$ precisely once.

The type of $L$ is the multiset $\left\{\left|S_{1}\right|,\left|S_{2}\right|, \ldots,\left|S_{m}\right|\right\}$. If there are $u_{i} S_{j} \mathrm{~s}$ of cardinality $t_{i}, 1 \leq i \leq k$, we say $L$ has type $t_{1}^{u_{1}} t_{2}^{u_{2}} \cdots t_{k}^{u_{k}}$.

Suppose $L$ and $M$ are a pair of partitioned incomplete Latin squares with partition $P . L$ and $M$ are called orthogonal if the array formed by the superposition of $L$ and $M, L \circ M$, contains every ordered pair in $S \times S-\bigcup_{i=1}^{m}\left(S_{i} \times S_{i}\right)$ precisely once. A set of $n$ partitioned incomplete Latin squares with partition $P$ is called a set of $n$ mutually orthogonal 
partitioned incomplete Latin squares of type $\left\{\left|S_{1}\right|,\left|S_{2}\right|, \ldots,\left|S_{m}\right|\right\}$ if each pair of squares is orthogonal.

We are now in a position to describe our first frame construction for balanced tournament designs with $A O R \mathrm{~s}$. This construction is a modification of the frame constructions that we used in $[8,9]$ for partitioned balanced tournament designs. We state it in the most general form.

THEOREM 3.1. If there exists a complementary $\left\{V_{1}, V_{2}, \ldots, V_{m}\right\}$-frame with property $T$, a set of three mutually orthogonal partitioned incomplete Latin squares of type $\left\{\left|V_{1}\right|,\left|V_{2}\right|, \ldots,\left|V_{m}\right|\right\}$ and side $\sum_{i=1}^{m}\left|V_{i}\right|$, and $B T D\left(\left|V_{i}\right|\right.$ $+1)$ with $A O R$ s for $1 \leq i \leq m$, then there is a $B T D\left(\sum_{i=1}^{m}\left|V_{i}\right|+1\right)$ with AORs.

Proof. Let $V=\bigcup_{i=1}^{m} V_{i}$ and let $W=\bigcup_{i=1}^{m} W_{i}$ where $\left|V_{i}\right|=\left|W_{i}\right|$ for $i=1,2, \ldots, m$.

Let $F$ be a complementary $\left\{V_{1}, V_{2}, \ldots, V_{m}\right\}$-frame with Property T. Let $\bar{F}$ be a complement of $F$ defined on $W$ so that $\bar{F}$ is a $\left\{W_{1}, W_{2}, \ldots, W_{m}\right\}$ frame. $A$ will denote the array formed by superimposing $F$ and $\bar{F}, A=$ $F \circ \bar{F}$.

Let $L_{1}$ and $L_{2}$ be a pair of orthogonal partitioned incomplete Latin squares of type $\left\{\left|V_{1}\right|,\left|V_{2}\right|, \ldots,\left|V_{m}\right|\right\}$ where $L_{1}$ has partition $\left\{V_{1}, V_{2}, \ldots\right.$, $\left.V_{m}\right\}$ and $L_{2}$ has partition $\left\{W_{1}, W_{2}, \ldots, W_{m}\right\}$. $L$ will be the array of pairs formed by superimposing $L_{1}$ and $L_{2}, L=L_{1} \circ L_{2}$.

Let $B_{i}$ be a $B T D\left(\left|V_{i}\right|+1\right)$ defined on $V_{i} \cup W_{i} \cup\{\alpha, \infty\} . B_{i}$ can be written in the following form.

$$
B_{i}=\begin{array}{|l|l|l|}
\hline A_{i} & D_{i} & C_{i} \\
\hline R_{i 1} & R_{i 2} & \{\alpha, \infty\} \\
\hline
\end{array}
$$

where $C_{i}$ is the common resolution class for the pair of almost orthogonal resolutions and $A_{i}$ and $D_{i}$ are $\left|V_{i}\right| \times\left|V_{i}\right|$ arrays.

We construct a $B T D$ on $V \cup W \cup\{\alpha, \infty\}$ as follows. Place the array $A_{i}$ in the hole of $A$ indexed by $V_{i}$ and $W_{i}$ and place the array $D_{i}$ in the hole of $L$ indexed by $V_{i}$ and $W_{i}$. Let $A^{\prime}$ and $D^{\prime}$ denote the resulting arrays. Add a new row $\left[R_{11} R_{21} \cdots \dot{R}_{m 1} R_{21} R_{22} \cdots R_{m 2}\right]$ to the array $\left[A^{\prime} L^{\prime}\right]$. Finally, add a new column $\left[C_{1} C_{2} \cdots C_{m}\{\alpha, \infty\}\right]^{T}$ to this array. It is straightforward to verify that the resulting array $B$ is a $B T D\left(\sum_{i=1}^{m}\left|V_{i}\right|+1\right)$ defined on $V \cup W \cup\{\alpha, \infty\}$.

One resolution of $B$ is given by the columns of $B$. To construct an almost orthogonal resolution, we use the transversals of $A$ (Property $\mathrm{T}$ ) and the transversals of $L$ provided by the third orthogonal partitioned incomplete 
Latin square together with the almost orthogonal resolutions of the $B_{i} \mathrm{~s}$. The common resolution class will be the last column of $B$.

Our second frame construction for balanced tournament designs with $A O R \mathrm{~s}$ is a modification of the standard frame construction for Howell designs. This construction produces $H(2 n, 2 n+2)$ s with Property T2.

THEOREM 3.2. If there exists $a\left\{V_{1}, V_{2}, \ldots, V_{m}\right\}$-frame with Property T2 where $\left|V_{i}\right| \equiv 0(\bmod 2)$ for $i=1,2, \ldots, m$, and if there exist $H\left(\left|V_{i}\right|,\left|V_{i}\right|+2\right)$ with Property T2 for $i=1,2, \ldots, m$, then there is an $H\left(\sum_{i=1}^{m}\left|V_{i}\right|, \sum_{i=1}^{m}\left|V_{i}\right|+2\right)$ with Property T2.

Proof. Let $F=\bigcup_{i=1}^{m} V_{i}$ and $\left|V_{i}\right|=2 t_{i}$ for $i=1,2, \ldots, m$. Let $F$ be a $\left\{V_{1}, V_{2}, \ldots, V_{m}\right\}$-frame with Property T2. Suppose the transversals associated with $F_{i}$ are $T_{1}^{i}, T_{2}^{i}, \ldots, T_{t_{i}}^{i}$.

Let $H_{i}$ denote an $H\left(\left|V_{i}\right|,\left|V_{i}\right|+2\right)$ with Property T2 defined on $V_{i} \cup$ $\{\alpha, \infty\}$ where $\{\alpha, \infty\}$ does not occur in $H_{i}$. The transversals of $H_{i}$ provided by Property T2 are $H_{1}^{i}, H_{2}^{i}, \ldots, H_{t_{i}}^{i},, H_{*}^{i}$ where $H_{*}^{i}$ contains every element of $V_{i}$ twice.

To construct an $H\left(\sum_{i=1}^{m}\left|V_{i}\right|, \sum_{i=1}^{m}\left|V_{i}\right|+2\right)$ defined on $V \cup\{\alpha, \infty\}$ and denoted by $H$, we place the array $H_{i}$ in the hole $F_{i}$ of $F$. We associate $t_{i}$ transversals of $H$ with this hole; they are $\tau_{\sum_{l=1}^{i-1} t_{t}+j}=H_{j}^{i} \cup T_{j}^{i}$ for $j=$ $1,2, \ldots, t_{i}$. This provides $\sum_{i=1}^{m} t_{i}$ transversals. The last transversal $\tau_{\sum t_{i}+1}$ required for Property T2 is $\bigcup_{i=1}^{m} H_{*}^{i}$. Each of these $\sum_{i=1}^{m} t_{i}+1$ transversals contains every element of $V \cup\{\alpha, \infty\}$ at most twice. Suppose the elements which do not occur twice in $\tau_{i}$ are $u_{i 1}, v_{i 1}, u_{i 2}, v_{i 2}$. Then, since each of the $H_{i}$ s has Property T2, the pairs $\left\{u_{i 1}, v_{i 1}\right\}$ do not occur in $H$ and $\bigcup_{i=1}^{\sum_{t} t_{j}+1}\left\{u_{i 2}, v_{i 2}\right\}=V \cup\{\alpha, \infty\}$. This verifies that $H$ has Property T2.

In Section 5, we use the existence results for frames from the next section together with Theorems 3.1 and 3.2 to construct several infinite classes of balanced tournament designs with $A O R$ s.

\section{Complementary frames with Property $T$ and frames with Property T2}

In the previous section, we described constructions for balanced tournament designs with $A O R \mathrm{~s}$ which require the existence of complementary frames with Property $T$ and frames with Property T2. Thus, to construct $B T D(n) \mathrm{s}$ with $A O R \mathrm{~s}$ we will construct frames and complementary frames 
with the appropriate properties. We can use 3-dimensional frames to construct some of these.

An $n$-dimensional $\left\{G_{1}, G_{2}, \ldots, G_{m}\right\}$-frame is an $n$-dimensional cube of side $\sum_{i=1}^{m}\left|G_{i}\right|$ such that each 2 -dimensional projection is a $\left\{G_{1}, G_{2}, \ldots\right.$, $\left.G_{m}\right\}$-frame.

An easy way to construct a complementary frame with Property $T$ is to construct a skew frame with a set of skew transversals. This is equivalent to constructing a 3-dimensional frame where the 2-dimensional projections are complementary frames with some additional structure [13]. Similarly, we can use 3-dimensional frames to construct frames with Property T2. Let $F$ be a $\left\{G_{1}, G_{2}, \ldots, G_{m}\right\}$-frame where $\left|G_{i}\right| \equiv 0(\bmod 2)$. We say $F$ is row complementary if at most one of the cells $(i, j)$ and $(i+1, j)$ is nonempty for $i \equiv 0(\bmod 2)$ and $0 \leq i \leq \sum_{i=1}^{m}\left|G_{i}\right|-1$ (that is, we could construct a complement for $F$ by interchanging pairs of rows). $R_{i}(F)$ will denote the collection of pairs in the $i$ th row of a row complementary frame $F$. Figure 8 contains an example of a row complementary frame of type $2^{5}$.

LEMMA 4.1. If there exists a 3-dimensional $\left\{G_{1}, G_{2}, \ldots, G_{m}\right\}$-frame with at least two row complementary $\left\{G_{1}, G_{2}, \ldots, G_{m}\right\}$-frames, $F_{1}$ and $F_{2}$, as 2dimensional projections where $R_{i}\left(F_{1}\right)=R_{i}\left(F_{2}\right)$ for $i=1,2, \ldots, \sum_{i=1}^{m}\left|G_{i}\right|$, then there is $a\left\{G_{1}, G_{2}, \ldots, G_{m}\right\}$-frame with Property $\mathrm{T} 2$.

Proof. Let $F$ be a 3-dimensional $\left\{G_{1}, G_{2}, \ldots, G_{m}\right\}$-frame where $\left|G_{i}\right|$ $=2 t_{i}$ for $i=1,2, \ldots, m$. Suppose $F_{1}$ and $F_{2}$ are two row complementary $\left\{G_{1}, G_{2}, \ldots, G_{m}\right\}$-frames which are 2-dimensional projections of $F$. Suppose $F_{3}$ is the remaining 2-dimensional projection of $F$. We show that $F_{3}$ is a $\left\{G_{1}, G_{2}, \ldots, G_{m}\right\}$-frame with Property T2. Let $R_{i}$ denote the collection of pairs in row $i$ of $F_{1}\left(\right.$ and $\left.F_{2}\right)$. Then $R_{i} \cup R_{i+1}$ for $i \equiv 0(\bmod 2)$ and $0 \leq i \leq \sum_{i=-1}^{m}\left|G_{i}\right|-1$ is a transversal of $F_{3}$ with the required property. For example, if $i \equiv 0(\bmod 2)$ and $0 \leq i \leq 2 t_{1}$, then $R_{i} \cup R_{i+1}$ contains every element of $\sum_{i=1}^{m} G_{i}$ precisely twice.

The equivalence of the types of frames that we are interested in to 3dimensional frames with special properties allows us to use $P B D$-closure to construct infinite classes of these frames. Definitions and results on $P B D$ closure can be found in [2]. Let $t$ be a positive integer. We define $S_{t}=\{u \mid$ there exists a skew $t^{u}$ frame with a set of $t n$ skew transversals $\}$, and we define $R C_{t}=\left\{u \mid\right.$ there exists a 3-dimensional $t^{u}$ frame with at least two row complementary $t^{u}$ frames $F_{1}$ and $F_{2}$ as 2-dimensional projections and $R_{i}\left(F_{1}\right)=R_{i}\left(F_{2}\right)$ for $\left.i=1,2, \ldots, t u\right\}$.

LeMMA 4.2. Let $t$ be a positive integer. $S_{t}$ and $R C_{t}$ are $P B D$-closed. 
[15] Balanced tournament designs with almost orthogonal resolutions

\begin{tabular}{|c|c|c|c|c|c|c|c|c|c|}
\hline & & & & & & $\begin{array}{lll}1 & 0 \\
4 & 1\end{array}$ & $\begin{array}{ll}1 & 1 \\
4 & 0\end{array}$ & $\begin{array}{ll}3 & 0 \\
2 & 1\end{array}$ & $\begin{array}{ll}3 & 1 \\
2 & 0\end{array}$ \\
\hline & & $\begin{array}{l}20 \\
30 \\
\end{array}$ & $\begin{array}{ll}2 & 1 \\
3 & 1\end{array}$ & $\begin{array}{ll}4 & 1 \\
1 & 1\end{array}$ & $\begin{array}{l}40 \\
10\end{array}$ & & & & \\
\hline $\begin{array}{ll}4 & 0 \\
3 & 1\end{array}$ & $\begin{array}{ll}4 & 1 \\
3 & 0\end{array}$ & & & & & & & $\begin{array}{ll}2 & 0 \\
0 & 1\end{array}$ & $\begin{array}{ll}2 & 1 \\
0 & 0\end{array}$ \\
\hline & & & & $\begin{array}{l}30 \\
40\end{array}$ & $\begin{array}{ll} & 1 \\
4 & 1\end{array}$ & $\begin{array}{ll}0 & 1 \\
2 & 1\end{array}$ & $\begin{array}{l}00 \\
29\end{array}$ & & \\
\hline $\begin{array}{ll}3 & 0 \\
11 & \\
\end{array}$ & $\begin{array}{ll}3 & 1 \\
1 & 0 \\
\end{array}$ & $\begin{array}{lll}0 & 0 \\
4 & 1 \\
\end{array}$ & $\begin{array}{lll}0 & 1 \\
4 & 0 \\
\end{array}$ & & & & & & \\
\hline & & & & & & $\begin{array}{ll}4 & 0 \\
0 & 0\end{array}$ & $\begin{array}{lll}4 & 1 \\
0 & 1 \\
\end{array}$ & $\begin{array}{ll}1 & 1 \\
3 & 1 \\
\end{array}$ & $\begin{array}{l}10 \\
30\end{array}$ \\
\hline & & $\begin{array}{ll}4 & 0 \\
2 & 1\end{array}$ & 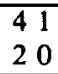 & $\begin{array}{ll}1 & 0 \\
0 & 1\end{array}$ & $\begin{array}{ll}1 & 1 \\
0 & 0\end{array}$ & & & & \\
\hline $\begin{array}{ll}2 & 1 \\
4 & 1\end{array}$ & $\begin{array}{l}20 \\
40\end{array}$ & & & & & & & $\begin{array}{l}00 \\
10\end{array}$ & $\begin{array}{ll}0 & 1 \\
1 & 1\end{array}$ \\
\hline & & & & $\begin{array}{l}00 \\
31 \\
\end{array}$ & $\begin{array}{ll}01 \\
30 \\
\end{array}$ & $\begin{array}{ll}2 & 0 \\
1 & 1 \\
\end{array}$ & $\begin{array}{ll}21 \\
10 \\
\end{array}$ & & \\
\hline $\begin{array}{ll}1 & 0 \\
2 & 0\end{array}$ & $\begin{array}{ll}1 & 1 \\
2 & 1\end{array}$ & $\begin{array}{ll}3 & 1 \\
0 & 1\end{array}$ & $\begin{array}{ll}3 & 0 \\
0 & 0\end{array}$ & & & & & & \\
\hline
\end{tabular}

Figure 8. A row complementary frame of type $2^{5}$

Proof. This follows immediately from the $P B D$-closure of skew frames, row complementary frames, and 3-dimensional frames [2, 18].

Before we describe constructions and existence results for complementary frames with Property $T$ and frames with Property T2, we note that some of the product constructions for frames can be generalized for these types of frames. Since these are standard constructions, we omit the proofs.

THEOREM 4.3. If there exists a complementary $t_{1}^{u_{1}} t_{2}^{u_{2}} \cdots t_{k}^{u_{k}}$ frame with Property $\mathrm{T}$ (or a $t_{1}^{u_{1}} \cdots t_{k}^{u_{k}}$ frame with Property T2), then there exists a complementary $\left(t_{1} x\right)^{u_{1}} \cdots\left(t_{k} x\right)^{u_{k}}$ frame with Property $\mathrm{T}$ (or a $\left(t_{1} x\right)^{u_{1}} \cdots\left(t_{k} x\right)^{u_{k}}$ frame with Property T2) for $x$ a positive integer and $x \neq 2,6$ or 10 .

The next two product constructions are direct generalizations of [18, Theorems 3.5 and 3.6].

THEOREM 4.4. Let $s=u(v-1)+1$, and let $t$ be a rational number such that $k t$ and $(v-1) / t$ are both integers and $(v-1) / t \neq 2,6$ or 10. If there exist complementary frames with Property $\mathrm{T}$ (or frames with Property T2) of type $(k t)^{u}$ and $k^{v}$, then there exists a complementary frame with Property $\mathrm{T}$ (or a frame with Property T2) of type $k^{s}$.

THEOREM 4.5. Let $s=u v$, and let $t$ be a rational number such that $k t$ and $v / t$ are both integers and $v / t \neq 2,6$ or 10 . If there exist complementary 
frames with Property T (or frames with Property T2) of type $(k t)^{u}$ and $k^{v}$, then there is a complementary frame with Property $\mathrm{T}$ (or a frame with Property T2) of type $k^{s}$.

In [13], we investigated the existence of skew frames with skew transversals. The following theorem summarizes these results. We note that the result for $u \equiv 1(\bmod 4)$ in [13] can be improved. We write $57=7(9-1)+1$ and $93=23(5-1)+1$ and apply Theorem 4.4 with $t=1 / 2$ to construct skew frames of type $2^{57}$ and $2^{93}$ with skew transversals.

THEOREM 4.6 [13]. (1) Let $n$ be an odd prime power, $n \geq 7, n \neq 9$. There exists a skew $1^{n}$ frame with a set of $n$ skew transversals. (2) Let $n \equiv 1(\bmod 2), n \geq 7$, and $n \neq 3 m$ where $m \geq 5$ and $(m, 3) \leq 1$. There exists a skew $1^{n}$ frame with a set of $n$ skew transversals. (3) Let $u \equiv 1$ $(\bmod 4), u \neq 33$ or 133 . There exists a skew frame of type $\left(2^{n}\right)^{u}$ with a set of $2^{n} u$ skew transversals for $n$ a positive integer.

We now consider the existence of frames with Property T2. The starteradder construction which was used in [13] to produce skew frames of type $\left(2^{n}\right)^{q}$ with sets of skew transversals where $q$ is a prime power and $q \equiv 1$ $(\bmod 4)$ can also be used to construct frames of type $\left(2^{n}\right)^{q}$ with Property T2. This is done by pairing the starters and adders for $i$ and $i+1(i \equiv 0$ $(\bmod 2))$. This construction also produces 3-dimensional frames with two row-complementary frames as 2-dimensional projections with the appropriate rows. We state this result in terms of 3-dimensional frames, since we will use it in a $P B D$-construction.

TheOReM 4.7. Let $n$ be a positive integer, and let $q \equiv 1(\bmod 4)$ be a prime power. There exists a 3-dimensional frame of type $\left(2^{n}\right)^{q}$ with two row-complementary frames, $F_{1}$ and $F_{2}$, as 2-dimensional projections where $R_{i}\left(F_{1}\right)=R_{i}\left(F_{2}\right)$ for $i=1,2, \ldots, 2^{n} q$.

Using $P B D$-closure as in $[13,18]$ and Theorem 4.4 , we also have the following.

THEOREM 4.8. Let $n$ be a positive integer. If $u \equiv 1(\bmod 4)$ and $u \neq$ 33 or 133, then there exists a 3-dimensional frame of type $\left(2^{n}\right)^{u}$ with two row-complementary frames, $F_{1}$ and $F_{2}$, as 2-dimensional projections with $R_{i}\left(F_{1}\right)=R_{i}\left(F_{2}\right)$ for $i=1,2, \ldots, 2^{n} u$.

The next construction uses complementary frames with Property $T$ to construct frames with Property T2. 
THEOREM 4.9. If there exists a complementary frame of type $t^{u}$ with Property $\mathrm{T}$ and a set of three mutually orthogonal partitioned incomplete Latin squares of type $t^{u}$, then there is a frame of type $(2 t)^{u}$ with Property T2. Furthermore, the frame of type $(2 t)^{u}$ with Property T2 is equivalent to a 3dimensional $(2 t)^{u}$ frame with two row complementary $(2 t)^{u}$ frames, $F_{1}$ and $F_{2}$, as 2-dimensional projections where $R_{i}\left(F_{1}\right)=R_{i}\left(F_{2}\right)$ for $1 \leq i \leq 2 t u$.

Proof. Let $V=\bigcup_{i=1}^{u} V_{i}$ and let $W=\bigcup_{i=1}^{u} W_{i}$. Let $F$ be a complementary $\left\{V_{1}, V_{2}, \ldots, V_{u}\right\}$-frame of type $t^{u}$ with Property T. $\bar{F}$ will be a complement of $F$ defined on $W$ so that $\bar{F}$ is a $\left\{W_{1}, W_{2}, \ldots, W_{u}\right\}$-frame of type $t^{u}$. $A$ will denote the array of pairs formed by the superposition of $F$ and $\bar{F}, A=F \circ \bar{F}$. Property $\mathrm{T}$ provides a set $\tau$ of $t u$ transversals of $A, \tau=\left\{T_{j}^{i} \mid i=1,2, \ldots, u, j=1,2, \ldots, t\right\}$.

Let $L_{1}$ and $L_{2}$ be a pair of OPILs of type $t^{u}$ where $L_{1}$ has partition $\left\{V_{1}, V_{2}, \ldots, V_{u}\right\}$ and $L_{2}$ has partition $\left\{W_{1}, W_{2}, \ldots, W_{u}\right\} . L$ will denote the array of pairs formed by the superposition of $L_{1}$ and $L_{2}, L=L_{1} \circ L_{2}$. We use the third OPIL of type $t^{u}$ to determine a set $S$ of $t u$ transversals of $L, S=\left\{S_{j}^{i} \mid i=1,2, \ldots, u, j=1,2, \ldots, t\right\}$ where $S_{j}^{i}$ contains $t$ cells from the $i$ th hole.

The following array $B$ is a frame of type $(2 t)^{u}$ defined on $V \cup W . B=$ $\left[\begin{array}{ll}A & 0 \\ 0 & L\end{array}\right]$ where 0 is a $t u \times t u$ empty array. The transversals for Property T2 are $T_{j}^{i} \cup S_{j}^{i}$ for $i=1,2, \ldots, u$ and $j=1,2, \ldots, t$.

Let $D_{i}$ denote the collection of pairs in row $i$ of $B$ and let $C_{i}$ denote the collection of pairs in column $i$ of $B$. let $k=0,1, \ldots, u-1$ and let $j=1,3, \ldots, 2 t-1$. Define $R_{2 k t+j}=T_{(j+1) / 2}^{k+1}$ and $R_{2 k t+j+1}=S_{(j+1) / 2}^{k+1}$.

We construct a row complementary $(2 t)^{u}$ frame as follows. Index of rows of a $2 t u$ square array with $R_{1}, R_{2}, \ldots, R_{2 t u}$ and the columns of the array with $D_{1}, D_{2}, \ldots, D_{2 t u}$. In cell $(i, j)$, place $R_{i} \cap D_{j}$. It is straightforward to verify that the resulting array $F_{1}$ is a row complementary $\left\{V_{1} \cup\right.$ $\left.W_{1}, \ldots, V_{u} \cup W_{u}\right\}$-frame. Similarly, we can construct a row complementary $\left\{V_{1} \cup W_{1}, \ldots, V_{u} \cup W_{u}\right\}$-frame using $R_{1}, \ldots, R_{2 t u}$ and $C_{1}, C_{2}, \ldots, C_{2 t u}$. It is clear that $R_{i}\left(F_{1}\right)=R_{i}\left(F_{2}\right) . \quad B$ (with a suitable permutation of the rows and columns), $F_{1}$ and $F_{2}$ are the three 2-dimensional projections of a 3-dimensional $\left\{V_{1} \cup W_{1}, \ldots, V_{u} \cup W_{u}\right\}$-frame of type $(2 t)^{u}$.

COROLlaRY 4.10. Let $n$ be an odd prime power, $n \geq 7, n \neq 9$. There is a 3-dimensional $2^{n}$ frame with two row complementary $2^{n}$ frames, $F_{1}$ and $F_{2}$, as 2-dimensional projections where $R_{i}\left(F_{1}\right)=R_{i}\left(F_{2}\right)$ for $i=1,2, \ldots, 2 n$.

Proof. We apply Theorem 4.9 using Theorem 4.6(1) and the existence of three $O P I L \mathrm{~s}$ of type $1^{n}$ for $n$ a prime power. 
We will now use the $P B D$-closure of $R C_{t}$ and a recent result of Mullin and Stinson [14] on pairwise balanced designs with odd block size to construct frames of type $2^{n}$ (for $n$ odd) with Property T2. $P_{5}$ will denote the set of odd prime powers greater than or equal to 5 and we denote $B\left(P_{5}\right)=$ $\left\{v \mid\right.$ there exists a $\left.\left(v, P_{5}\right)-P B D\right\}$. Let $Q=\{15,33,39,51,75,87,93$, $183,219\}$

THEOREM 4.11 [14]. If $n \geq 5$ is odd and $n \notin Q$, then $n \in B\left(P_{5}\right)$.

The next result follows immediately from the $P B D$-closure of $R C_{2}$, Lemma 4.1 and Theorem 4.11 together with Corollary 4.10 , and Theorem 4.8.

THEOREM 4.12. If $n \geq 5$ is odd and $n \notin Q$, then there is a $2^{n}$ frame with Property $\mathrm{T} 2$.

\section{Applications}

In this section, we use the results of the previous sections to provide several infinite classes of balanced tournament designs with $A O R \mathrm{~s}$. We recall that we have constructed $B T D(n)$ with $A O R$ s for $n=3,5$ and 6 (Section 2). These are respectively $H(4,6), H(8,10)$, and $H(10,12)$ with Property T2. We will use these small designs in our recursive constructions. We first consider applications of Theorem 3.1.

THEOREM 5.1. Let $n$ be a positive integer, $n \neq 8$ or 33. There exists $a$ $B T D(m)$ with AOR $s$ for $m=8 n+3$ and $m=16 n+5$.

Proof. (i) Let $m=8 n+3$. We apply Theorem 3.1 using complementary frames of type $2^{4 n+1}$ with Property $\mathrm{T}$ and three $O P I L$ s of type $2^{4 n+1}$, as well as a $B T D(3)$ with $A O R \mathrm{~s}$. The existence of complementary frames of type $2^{4 n+1}$ with Property $T$ is provided by Theorem 4.8. The existence of three $O P I L$ s of type $2^{4 n+1}$ is proved in [19].

(ii) Let $m=16 m+5$. We apply Theorem 3.1 using complementary frames of type $4^{4 n+1}$ with Property T (Theorem 4.8), three OPILs of type $4^{4 n+1}$ and a $B T D(5)$. The three $O P I L \mathrm{~s}$ of type $4^{4 n+1}$ can be constructed using the direct product for $O P I L \mathrm{~s}$.

Next, we apply Theorem 3.2, which constructs Howell designs with Property $\mathrm{T} 2$. 
THEOREM 5.2. Let $n$ be a positive integer, $n \geq 2$, and $2 n+1 \notin Q$. There exists a $B T D(m)$ with AOR sor $m=8 n+5$ and $m=10 n+6$.

Proof. (i) Let $m=8 n+5$. We apply Theorem 3.2 using a frame of type $8^{2 n+1}$ with Property T2 and an $H(8,10)$ with Property T2. The frame of type $8^{2 n+1}$ with Property T2 can be constructed by using the frames of type $2^{2 n+1}$ with Property T2 provided by Theorem 4.12 and Theorem 4.3 with $x=4$.

(ii) Let $m=10 n+6$. A frame of type $10^{2 n+1}$ with Property T2 can be constructed using Theorem 4.12 and Theorem 4.3 with $x=5$. Again we apply Theorem 3.2 , using an $H(10,12)$ in the holes of the $10^{2 n+1}$ frame.

We can also use Howell designs to construct two other classes of $B T D(m)$ s with $A O R \mathrm{~s}$, where $m \equiv 1(\bmod 4)$.

THEOREM 5.3. Let $n$ be a positive integer. There exist $B T D(m)$ with $A O R$ s for $m=20 n+1$ and $m=20 n+5$.

Proof. It is well known that there exist $(v, 5,1)-B I B D \mathrm{~s}$ for $v \equiv 1$ and $5(\bmod 20), v \geq 5$ [4]. Let $v=20 n+1$ or $20 n+5$. By deleting one element from each of these designs, we can construct $G D D(20 n ; 5 ; 4 ; 0,1)$ and $G D D(20 n+4 ; 5 ; 4 ; 0,1)$ respectively. To each of these designs, we apply Wilson's Fundamental Construction [22], weighting by a factor of 2 and replacing each block with the $2^{5}$ frame with Property T2 from Theorem 4.7, to construct frames with Property T2 of type $8^{5 n}$ and $8^{5 n+1}$. Then we apply Theorem 3.2 using $H(8,10)$ s with Property T2.

We note that the $B T D(n)$ that we constructed for $n=3,5$, and 6 have Property $C^{\prime}$. Since the recursive constructions that we have used in this section preserve Property $C^{\prime}$, it is straightforward to verify that the designs produced by Theorems 5.1, 5.2, and 5.3 all have Property $C^{\prime}$.

There are several other smaller classes of balanced tournament designs with $A O R \mathrm{~s}$ which can be constructed by applying Theorems 3.1 and 3.2 using the designs described above and frames with larger holes (which can be found using the product constructions). However, in order to complete the spectrum of balanced tournament designs with $A O R \mathrm{~s}$ using the frame constructions, we require the existence of several other frames with Property T2 or complementary frames with Property $\mathrm{T}$ and the appropriate set of $O P I L \mathrm{~s}$. For example, the existence of complementary frames of type $2^{n}$ with Property $\mathrm{T}$ for all $n \geq 5$ and a set of three $O P I L \mathrm{~s}$ of type $2^{n}$ would allow us to construct all but a small number of $B T D(m)$ where $m \equiv 1(\bmod 2)$. The existence of Howell designs was determined using frame constructions [17]. These constructions used non-uniform frames. All of our existence results 
in Section 4 are for uniform frames. Thus, we need to determine the existence of some non-uniform frames with Property T2, in order to use similar constructions to find the spectrum of $H(2 n, 2 n+2)$ with Property T2 (or $B T D(n+1)$ s with $A O R \mathrm{~s})$.

\section{Note added in proof}

A recent result by Lindner and Stinson can be used to generalize Theorem 5.3 to include almost all $m$ congruent to 1 mod 4 . In "Nesting of cycle systems of even length", Graphs and Combinatorics (submitted), they show that there is a $G D D(4 u ;\{5,9,13,17,29,49\} ; 4 ; 0,1)$ for all $u \geq 5$ and $u \notin N=\{7,8,12,14,18,19,23,23,33,34\}$. The construction of Theorem 5.3 then produces $B T D(m)$ with $A O R$ s for all $m=4 u+1, u \geq 5$ and $u \notin N$.

\section{References}

[1] B. A. Anderson, 'Howell designs of type $H(p-1, p+1)$ ', J. Combin. Theory Ser. A 24 (1978), 131-140.

[2] J. H. Dinitz and D. R. Stinson, 'The construction and uses of frames', Ars Combin. 10 (1980), 31-53.

[3] J. H. Dinitz and D. R. Stinson, 'MOLS with holes', Discrete Math. 44 (1983), 145-154.

[4] H. Hanani, 'On balanced incomplete block designs with blocks having 5 elements', $J$. Combin. Theory Ser. A 12 (1972), 184-201.

[5] S. H.Y. Hung and N. S. Mendelsohn, 'On Howell designs', J. Combin. Theory Ser. A 16 (1974), 174-198.

[6] E. R. Lamken, 'On classes of doubly resolvable $(v, 3,2)$-BIBDs from balanced tournament designs', Ars Combin. 24 (1987), 85-91.

[7] E. R. Lamken and S. A. Vanstone, 'The existence of factored balanced tournament designs', Ars Combin. 19 (1985), 157-160.

[8] E. R. Lamken and S. A. Vanstone, 'Partitioned balanced tournament designs of side $4 n+1$ ', Ars Combin. 20 (1985), 29-44.

[9] E. R. Lamken and S. A. Vanstone, 'The existence of partitioned balanced tournament designs of side 4n+3', Ann. Discrete Math. 34 (1987), 319-338.

[10] E. R. Lamken and S. A. Vanstone, 'The existence of partitioned balanced tournament designs', Ann. Discrete Math. 34 (1987), 339-352.

[11] E. R. Lamken and S. A. Vanstone, 'Balanced tournament designs and resolvable $(v, 3$, 2)- BIBD s', Discrete Math. (to appear).

[12] E. R. Lamken and S. A. Vanstone, 'Complementary Howell designs of side $2 n$ and order $2 n+2$ ', Congr. Numer. 41 (1984), 85-113.

[13] E. R. Lamken and S. A. Vanstone, 'Skew transversals in frames', J. Combin. Math. Combin. Comp. 2 (1987), 37-50.

[14] R. C. Mullin and D. R. Stinson, 'Holey SOLSSOMs', Utilitas Math. 25 (1984), 159-169. 
[15] A. Rosa and D. R. Stinson, 'One-factorizations of regular graphs and Howell designs of small order', Utilitas Math. 29 (1986), 99-124.

[16] R. J. Schellenberg, G. H. J. van Rees, and S. A. Vanstone, 'The existence of balanced tournament designs', Ars Combin. 3 (1977), 303-318.

[17] D. R. Stinson, Some classes of frames and the spectrum of skew room squares and Howell designs, (Ph.D. Thesis, University of Waterloo, 1981).

[18] D. R. Stinson, 'On the existence of skew Room frames of type $2^{n}$, Ars Combin. 24 (1987), 115-128.

[19] D. R. Stinson and L. Zhu, 'On sets of three MOLs with holes', Discrete Math. 54 (1985), 321-328.

[20] D. R. Stinson and L. Zhu, 'On the existence of MOLs with equal-sized holes', Aequationes Math. 33 (1987), 96-105.

[21] S. A. Vanstone, 'Doubly resolvable designs', Discrete Math 29 (1980), 77-86.

[22] R. M. Wilson, 'Constructions and uses of pairwise balanced designs', Math. Centre Tracts 55 (1974), 18-41.

Department of Combinatorics and Optimization

University of Waterloo

Waterloo, Ontario N2L 3G1

Canada 